EPJ Web of Conferences 37, 01015 (2012)

DOI: 10.1051/epjconf/20123701015

(C) Owned by the authors, published by EDP Sciences, 2012

\title{
The frontiers of the virtual photons program at MAMI
}

C. Sfienti ${ }^{1, \text { a }}$, P. Achenbach ${ }^{1}$, C. Ayerbe Gayoso ${ }^{1}$, R. Böhm $^{1}$, D. Bosnar ${ }^{2}$, L. Debenjak ${ }^{3}$, A. Denig ${ }^{1}$, M. O. Distler ${ }^{1}$, A. Esser ${ }^{1}$, H. Fonvieille ${ }^{4}$, I. Friščič ${ }^{2}$, Y. Fujii ${ }^{5}$, T. Gogami ${ }^{5}$, Mar Gòmez Rodríguez ${ }^{1}$, H Hashimoto $^{5, \mathrm{~b}}$, S. Hirose ${ }^{5}$, H. Merkel ${ }^{1}$, D. G. Middleton ${ }^{1}$, M. Molitor ${ }^{1}$, U. Müller ${ }^{1}$, L. Nungesser ${ }^{1}$, S. Nagao ${ }^{5}$, S. N. Nakamura ${ }^{5}$, J. Pochodzalla ${ }^{1}$, T. Saito ${ }^{1}$, S. Sànchez Majos ${ }^{1}$, A. Sanchez Lorente ${ }^{1}$,

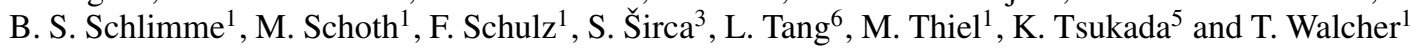

1 Institut für Kernphysik, Johannes Gutenberg-Universität D-55099 Mainz, Germany

2 Department of Physics, University of Zagreb, HR-10002 Zagreb, Croatia

3 University of Ljubljana and Institut "Jožef Stefan", SI-1000 Ljubljana, Slovenia

${ }^{4}$ Laboratoire de Physique Corpusculaire de Clermont-Ferrand, CNRS/IN2P3, Universitè Blaise Pascal, F-63000 Clermont-Ferrand, France

5 Department of Physics, Tohoku University, Sendai, Japan

6 Department of Physics, Hampton University, Hampton, VA USA

\begin{abstract}
The most recent results and the future physics program of the high precision electron-scattering experiment at MAMI are briefly outlined.

The A1 high-resolution spectrometers facility allows for a unique quality of virtual photon experiments. High precision form factor measurements, few-baryon systems highresolution structure studies and the innovative way in the search of dark photons illustrate the interplay between such diverse fields as precision atomic physics, nuclear astrophysics and astroparticle physics, where hadron physics plays a central and connecting role.
\end{abstract}

\section{Introduction}

The ultimate goal of strong-interaction physics is to provide a unifying picture of the structure of hadrons, their mutual interactions and the properties of nuclei from Quatum Chromo Dynamics (QCD). Much progress has been made in the last few years.

Presently, our knowledge of the subatomic world is expanding towards different frontiers. The highenergy frontier, probed at the LHC at CERN, is driven by directly producing new particles and uncovering their interactions. Truly complementary a vast discovery potential for new physics involves the comparison of precision measurements with theoretical predictions. Prominent examples of this interplay at the precision frontier are the ultra-high precision atomic physics measurements in electronic atoms. Pushing this precision frontier reveals a new frontier determined by our present understanding of hadronic physics at low energies. The frontier at low energies is defined by our quantitative understanding of QCD. The exploration of the full potential of electroweak probes at these energies is the focus of the experimental program at the MAMI accelerator.

The Mainz Microtron MAMI [1] is a continuous-wave electron accelerator operated at the Institute for Nuclear Physics at the University of Mainz. MAMI delivers electron beams with energies up to $1.6 \mathrm{GeV}$, currents of up to $100 \mu \mathrm{A}$ unpolarised or $40 \mu \mathrm{A}$ with $85 \%$ polarisation and an excellent beam quality for precision experiments. Over the past decade, the yearly hours of operation were reaching or even exceeding 6000, underlining the extreme high reliability of the installation. The accelerator is complemented with three major detector setups for precision scattering experiments mostly with

\footnotetext{
a e-mail: sfienti@kph.uni-mainz.de

b deceased
} 
polarized electrons and photons.

The most prominent facility is the A1 three-spectrometer set-up [2], which is optimized for precision coincidence measurements of charged particles in exclusive electron scattering experiments, with a momentum resolution of $\Delta p / p \approx 10^{-4}$, a solid angle acceptance of up to $28 \mathrm{msr}$, and momentum acceptance of up to $25 \%$. For out-of-plane measurements, one of the spectrometer can be inclined to angles of up to $10^{\circ}$. A proton recoil polarimeter allows for double polarisation measurements if combined with beam or target polarization. In addition, a short-orbit spectrometer with $1.5 \mathrm{~m}$ path length for detection of low-momentum pions and a kaon spectrometer KaOs capable of measuring high-momentum particles at small scattering angles are available. The setup can be further completed by neutron detectors or a neutron recoil polarimeter.

A few selected physics topics of the experimental program on electroproduction will be outlined in the following, to show the interplay between low energy hadron physics and atomic, nuclear and beyondthe-Standard-Model particle physics.

\section{Form factor measurements}

The form factor of a baryon is one of the most fundamental observables in hadron physics. It is directly related to the distribution of charge and magnetization of the baryon and through such imaging provides the basis of nearly all studies of the hadron structure. Form factors become a more and more important input also for other precision experiments in several fields of physics. Well known examples are the hydrogen Lamb shift and the hydrogen hyperfine splitting.

In the year 2010 two groups reported on new measurements of the proton charge radius. One determination resulted from a Lamb shift measurement in muonic hydrogen $(\mu p)$ and a proton radius of $r_{p}=0.84184(67) \mathrm{fm}$ has been derived, 10 times more precise as previously known [3] and about $4 \%$ or five standard deviations smaller than previously established.

The second experiment has been performed at MAMI [4]. Precise results of the elastic electron-proton scattering cross section were obtained in a measurement in about 1400 different kinematical settings, with negative four-momentum transfers squared from $Q^{2}=0.004$ to $1(\mathrm{GeV} / \mathrm{c})^{2}$. Statistical errors are below $0.2 \%$, and great effort was spent to reduce systematic errors down to the $1 \%$ level. The electric and magnetic form factors of the proton were extracted by fits of a large variety of form factor models directly to the cross sections, and they are depicted in Fig. 1 This new method makes use of the full kinematic range of the experimental data for the determination of the form factors instead of using in the fit, as traditionally done with the Rosenbluth separation, only data measured at a fixed $Q^{2}$.

The charge and magnetic radii are determined to be $\left\langle r_{E}^{2}\right\rangle^{1 / 2}=0.879(5)_{\text {stat }}(4)_{\text {syst }}(2)_{\text {model }}(4)_{\text {group }}$ fm and $\left\langle r_{M}^{2}>^{1 / 2}=0.777(13)_{\text {stat }}(9)_{\text {syst }}(5)_{\text {model }}(2)_{\text {group }} \mathrm{fm}\right.$. The MAMI result confirmed the results obtained from $\mathrm{H}$ spectroscopy [5] and also the value obtained from a global fit to previous e-p scattering data [6].

Several attempts to understand the disagreement of the proton radius values extracted from the various experiments ( $\mu$ p, $\mathrm{H}$ and e-p scattering) have made since then. Nevertheless, it is still unclear where the solution to the proton size puzzle lies.

Different strategies are therefore needed. On one side new measurements of the proton form factor are necessary. In particular new precision measurements of the proton form factor at both low and high momentum transfers are planned at MAMI. At low momentum transfer the determination of the proton radius below the $1 \%$ level is aimed at the new technique of initial state radiation. Detailed simulations showed already, that statistical accuracy in the proton charge radius of better than $0.1 \%$ can be easily achieved. So the determination of the radius is limited by the systematic error. The technique of initial state radiation promises to dramatically reduce the experimental systematic error.

At higher momentum transfers, we plan to continue the precision Rosenbluth measurements, which were limited by the endpoint energy of MAMI B, to the range accessible now by MAMI C. The aim is to have precision form factor data up to $Q^{2}=1.5(\mathrm{GeV} / \mathrm{c})^{2}$ to have a consistent connection with higher energy experiments, e.g. at JLab. The experimental technique is established and well understood.

On the other side, since in the near future measurements of the Lamb shift in light muonic atoms will take place with unequaled precision, a campaign of measurements is also planned to measure the form 

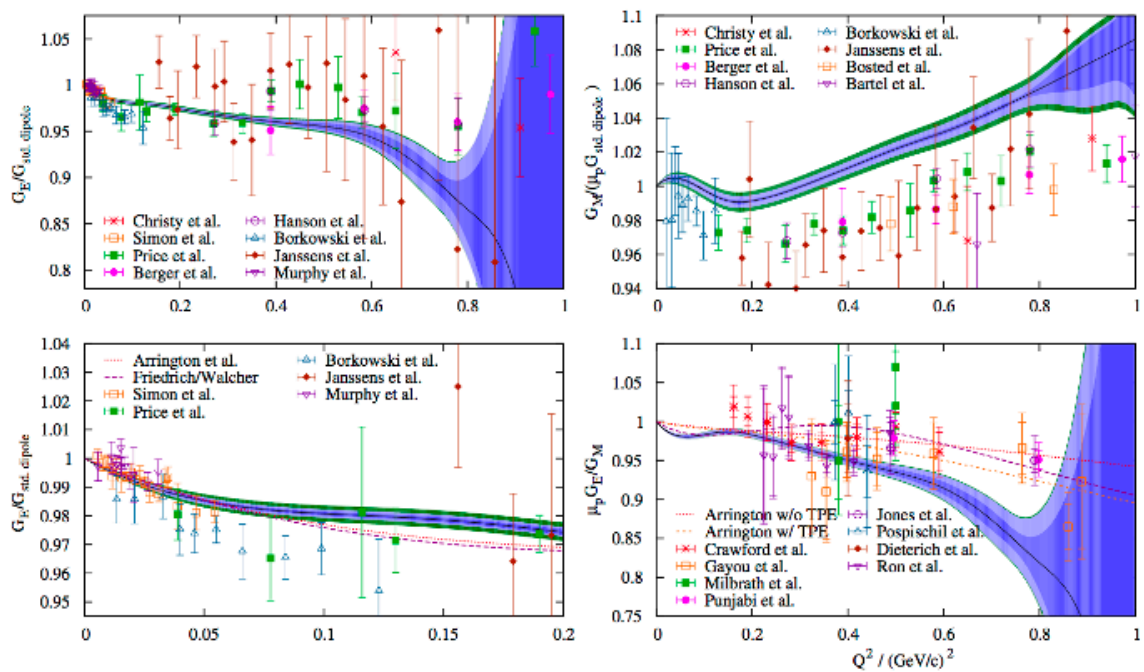

Fig. 1. The form factors $G_{E}$ and $G_{M}$ normalised to the standard dipole and $G_{E} / G_{M}$ as a function of $Q^{2}$. Black line: best fit to the data. The error bands, ordered from innermost to outermost, indicate the statistical $68 \%$ pointwise confidence band, the experimental systematic error, and the variation of the Coulomb correction by $\pm 50 \%$, respectively. The different data points depict previous measurements and dashed lines are previous fits to the old data (see Ref. [4] and references therein).

factor of deuterium and ${ }^{3} \mathrm{He}$ with high precision over a large momentum transfer range. The derived radii and other moments of the charge distribution will be crucial for the interpretation of future Lamb shift measurement on muonic deuterium and muonic helium.

The domain of light nuclei is also the natural testing ground for linking nucleons and sub-nucleon degrees of freedom with complex nuclei. Today nuclear structure theory has started to explore the connection to QCD in a quantitative way. Promising candidates are chiral effective field theories $(\chi \mathrm{EFT})$, providing a link to QCD by obeying the relevant conservation laws and symmetries in the low energy domain of nuclear few-body systems [7].

Because of the relatively large value of the strange-quark mass the extension of $\chi \mathrm{EFT}$ into the SU(3) sector is challenging and far from solved. Experiments exploring the properties of hypernuclei and ultimately aiming at the determination of the hyperon-nucleon $(\mathrm{YN})$ and hyperon-hyperon (YY) interactions are of great importance in guiding current efforts.

\section{Precision spectroscopy of light hypernuclei}

The physics of strangeness in hadronic systems is a constantly developing field with a large variety of production reactions, making use of precision coincidence measurements [8].

Studies of $\Lambda$-hypernuclei has led to significant gain in knowledgein understanding the baryon strangeness in nuclear medium. In general one can distinguish between two classes of reactions to produce and identify hypernuclei. The first relies on the detection of the decay products, like $\gamma$-rays from excited states and $\pi^{-}$from weak decays. Alternatively one can employ quasi two-body kinematics to identify the produced hypernucleus by a missing-mass analysis of the incident beam and the observed associated meson.

A novel method of decay spectroscopy is the high resolution pion spectroscopy of weak decays with electron beams [9]. This unique experimental technique is based on the study of the precisely measured monochromatic pions from hypernuclear two body mesonic weak decay. While missing mass experiments are limited to nuclei close to the initial target the quasi-free kaon production of an excited primary hypernucleus and its subsequent decay gives access to a variety of light and exotic hypernu- 


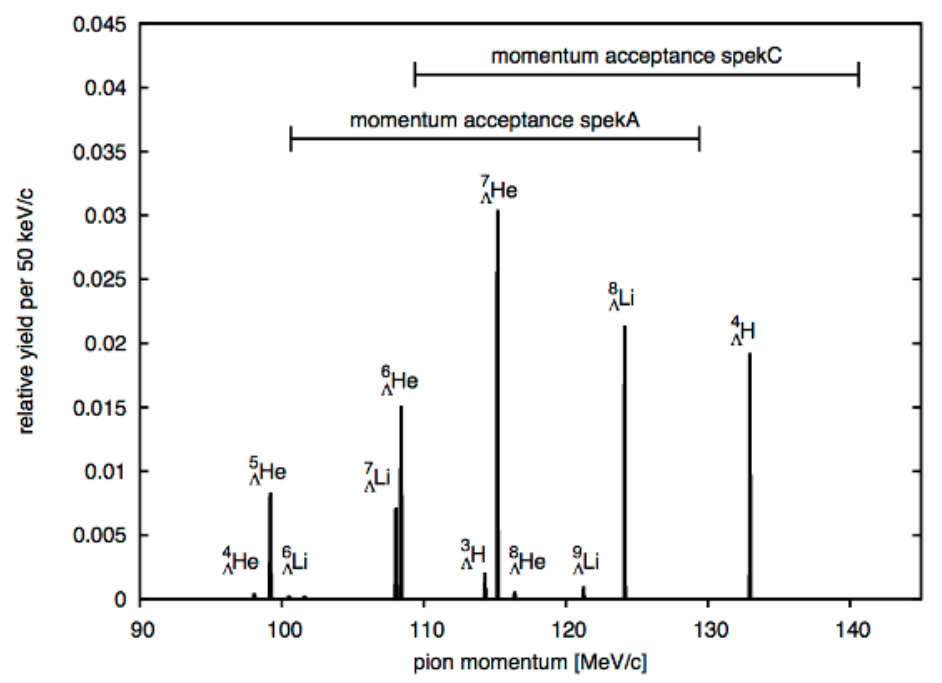

Fig. 2. Expected pion spectrum of the experiment performed at MAMI. Since the pions are produced by a two body decay with a long lifetime $(\approx 263 \mathrm{ps})$ narrow peaks are obtained. Their broadening arise from the variation of the energy loss of the low energetic pions inside the target foil. The relative fragmentation yields are taken from a statistical decay model [14]. The wide momentum acceptances of the high resolution spectrometers allows the detection of several different hypernuclei in one single experiment.

clei, some of which cannot be produced or measured precisely by other means.

Thus this novel technique can open a new era in the hypernuclear physics research allowing for the first time precision studies of the Charge Symmetry Breaking (CSB) in hypernuclei.

The FINUDA collaboration analyzed the existing $\mathrm{A}\left(\mathrm{K}_{\text {stop }}^{-}, \pi^{-}\right)_{Y} \mathrm{~A}$, data for two body $\pi^{-}$- decay and its spectroscopy. The results showed fragmentation production from primary hypernuclei and $\pi^{-}$- spectroscopy featured by the nature of the ground state of the decayed hypernuclei [10], thus providing an extremely encouraging demonstration of this technique.

A pioneering experiment for high resolution decay pion spectroscopy has been recently commissioned at the A1 spectrometer facility [11]. An instrument of central importance for this program is the newly installed magnetic double spectrometer Kaos [12]. Its compact design and its capability to detect simultaneously negative and positive charged particles up to the highest particle momenta significantly extends the capability of the spectrometer facility. The possibility to operate KAOs at $0^{\circ}$ makes it a unique instrument to also study elementary kaon production [13].

In the first pilot experiment, $K^{+} \Lambda$ pairs are produced on a ${ }^{9} \mathrm{Be}$ target by a $1.6 \mathrm{GeV}$ electron beam. By detecting the kaon, reactions involving strangeness production can be tagged with the Kaos spectrometer. The scattered electron from the reaction is disregarded.

With a certain probability the $\Lambda$ hyperon is captured by the remaining nucleus and forms a highly excited ${ }_{\Lambda}^{9} \mathrm{Li}$ hypernucleus, which due to its high excitation is likely to fragment into a lighter hyperfragment and one or more nucleons or light nuclei. Usually the newly formed hyperfragment deexcites electromagnetically to its ground state before its decay.

If a two body mesonic weak decay occurs, the pion momentum is measured with high resolution magnetic spectrometers. From the pion momentum the ground state mass of the hyperfragment and therefore the binding energy of the $\Lambda$ hyperon in the nucleus can be reconstructed. The expected resulting pion spectrum for this set-up is shown in figure 2 .

As the preliminary analysis of the pilot experiment showed [11], the main difficulty in the analysis of the data is the high flux of background particles in the KAos spectrometer, mostly positrons produced by pair production of bremsstrahlung photons in the target, but also pions and protons detected in the 
spectrometer.

One possibility to reduce this background is to use lead absorbers: simulations have shown that in the momentum range of 600 to $1200 \mathrm{MeV} / \mathrm{c}$ as a lead shield of $50 \mathrm{~mm}$ can absorb most of the positrons due to their creation of electromagnetic showers, while kaons in most cases are deflected only by a few degrees. With the improved setup data taking is taking place in fall 2012.

\section{Search for the dark photon}

An extra $U(1)$ boson of the dark matter sector is a possible explanation of several up to now unresolved experimental problems, e.g. the discrepency between experiment and Standard Model prediction for the anomalous magnetic moment of the muon. Recently, the focus of dark photon searches has moved to masses in the few $\mathrm{MeV}$ to $\mathrm{GeV}$ mass range. As Arkani-Hamed et al. in Ref. [15] has pointed out, dark photons with such masses and with relatively large couplings to ordinary matter, could explain several astronomical observations.

As discussed by Bjorken et al. [16], low-energy electron acclerators offer a highly-sensitive access to such searches. It turns out that indeed the MAMI facility with the high-resolution A1 spectrometers is ideally suited for such searches in the mass range above approximately $50 \mathrm{MeV}$. From a first pilot run, initially intended as a test beam experiment, a competitive exclusion limit for masses around 250 $\mathrm{MeV}$ could already be extracted [17] . The experiment was performed at an electron energy of 855
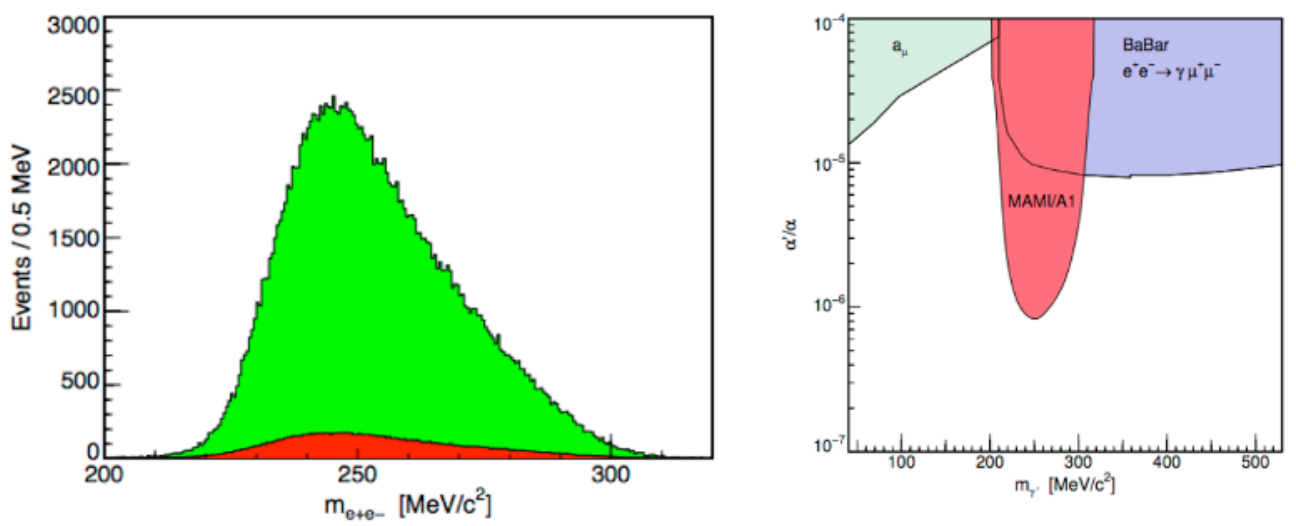

Fig. 3. Left Panel: Invariant distribution of the electron/positron pair. Right Panel: Exclusion limits for dark photons, in the plane mass versus coupling strength. Also shwon in the figure are the results obtained from the anomalous magnetic moment of the muon [18] and the BABAR results for the decay $e^{+} e^{-} \leftarrow \gamma \mu+\mu-[19]$

$\mathrm{MeV}$ on a Tantalum target to optimize the count rate. The scattered electron and the recoil nucleus are not detected. For the detection of the lepton pair, two high resolution spectrometers were used. The clear coincidence peak between the two spectrometers after cut on identified leptons, with a width of $1 \mathrm{~ns}$ (FWHM) has used as identified lepton pairs. For the events in the timing window, the invariant mass of the lepton pair was calculated using the full reconstructed four momenta of the spectrometers. Fig. 3 (left panel) shows the resulting missing mass distribution. The red shaded area illustrates the contribution of accidental coincidences.

A dark boson would show up in this spectrum as a sharp peak, with a width given by the experimental mass resolution. The expected mass resolution of less than $0.5 \mathrm{MeV} / c^{2}$ was determined by a simulation based on the measured angular and momentum resolution of the spectrometers.

Based on the invariant mass distribution of Fig. 3, an exclusion limit with a confidence limit of $90 \%$ was extracted. From the exclusion limit in terms of count rate, the exclusion limit in terms of the 
coupling is calculated by normalization to the measured QED background and the background by random coincidences. Fig. 3 (right panel) shows the resulting exclusion limit.

Based on the first experiment an extended experimental program at MAMI is currently under design.

\section{Conclusions and outlook}

The physics program at the A1 spectrometer facility at MAMI in the present day research environment lies in the interplay between such diverse fields as high-energy physics, precision atomic physics, and nuclear physics, where hadron physics plays a central and connecting role.

A precise understanding of hadron structure is of high relevance in the field of atomic physics, as demonstrated by the 5 standard deviation discrepancy of the charge radius of the proton extracted with different technique. Precise measurement of nucleon form factors at low $Q^{2}$ at MAMI are scheduled, with the aim to extract a proton radius with precision well below $1 \%$.

Advances in the domain of effective field theories in recent years have opened up a new era in the study of interactions in few-baryon systems. Precision pion spectroscopy of $\Lambda$ hypernuclei will allow to improve our knowledge of binding energies of single hypernuclei by one order of magnitude, allowing for the first time precision studies of charge symmetry breaking in hypernuclei.

The dark photon search has a discovery potential for beyond-the-Standard-Model particles. A unique chance will be given by the future MESA project, a dedicated low energy accelerator with very high beam intensity under construction at Mainz. It will allow accessing the region of lower masses for the dark photon searches, which cannot be reached by standard spectrometer setup due to the narrow cone of the decay leptons.

\section{Aknowledgements}

This work was supported in part by the Federal State of Rhineland-Palatinate and by the Deutsche Forschungsgemeinschaft with the Collaborative Research Center 1044.

\section{References}

1. A. Jankowiak, Eur. Phys. J A28, (2006) 149

2. K.I. Blomqvist et al., Nucl. Instrum. Meth. A403, (1998) 263

3. R. Pohl et al., Nature 466 (2010) 213

4. J. C. Bernauer et al. (A1 Collaboration), Phys. Rev. Lett. 105, (2010) 242001

5. P. J. Mohr, B. N. Taylor and D. B. Newell, Rev. Mod. Phys. 80, (2008) 633

6. P. G. Blunden and I. Sick, Phys. Rev. C 72, (2005) 057601

7. E. Epelbaum, Prog. Part. Nucl. Phys. 67, (2012) 343

8. O. Hashimoto and H. Tamura, Prog. Part. Nucl. Phys. 57, (2006) 564

9. L. Tang, Proceedings of the International Symposium, Strangeness in Nuclear and Hadronic Systems, (World Scientific, Sendai, 2008) page 346

10. E. Botta et al., Int. J. Mod. Phys. E 19, (2010) 2566

11. A. Esser et al. (A1 Collaboration), Proceedings of the 50th International Meeting on Nuclear Physics, (PoS, Bormio, 2012) page 038

12. P. Achenbach et al. (A1 Collaboration), Eur. Phys. J. ST 198, (2011) 307

13. P. Achenbach et al. (A1 Collaboration), this volume

14. A. Sanchez Lorente et al., Phys. Lett. B 697, (2011) 222

15. N. Arkani-Hamed, Phys. Rev. D 79, (2009) 015014

16. J. D. Bjorken et al., Phys. Rev. D 80, (2009) 075018

17. H. Merkel et al. (A1 Collaboration), Phys. Rev. Lett 106, (2011) 251802

18. G. W. Bennett et al., Phys. Rev. D 73, (2006) 072003

19. B. Aubet et al., Phys. Rev. Lett. 103, (2009) 081803 